\title{
Socioeconomic Factors Account for Variability in Language Skills in Preschoolers with Autism Spectrum Disorders
}

\author{
Lindsay Olson, MS ${ }^{*}$, , Mikaela Kinnear, PhD*, Bosi Chen, MS $^{*}{ }^{\dagger}$, Sarah Reynolds, BA*, \\ Cynthia Ibarra, MA ${ }^{*}$, Tiffany Wang, MA*, Annika Linke, PhD*, Inna Fishman, PhD ${ }^{*}$, \\ *Department of Psychology, Brain Development Imaging Laboratories, San Diego State \\ University, San Diego, CA \\ †San Diego State University/UC San Diego Joint Doctoral Program in Clinical Psychology, San \\ Diego, CA.
}

\section{Abstract}

Objective: Although no longer required for a diagnosis, language delays are extremely common in children diagnosed with autism spectrum disorders (ASD). Factors associated with socioeconomic status (SES) have broad-reaching impact on language development in early childhood. Despite recent advances in characterizing autism in early childhood, the relationship between SES and language development in ASD has not received much attention. The objective of this study was to examine whether toddlers and preschoolers with ASD from low-resource families are more likely to experience language delays above and beyond those associated with autism itself.

Methods: Developmental and diagnostic assessments including the Mullen Scales of Early Learning, the Autism Diagnostic Observation Schedule, Second Edition, and the Vineland Adaptive Behavior Scales were obtained from 62 young children with ASD and 45 typically developing children aged 15 to 64 months. Sociodemographic information including household income, maternal education, and racial/ethnic identity was obtained from caregivers. Multiple regression models were used to test for associations between socioeconomic indices and language scores.

Results: Maternal education accounted for variability in expressive language (EL) and receptive language (RL), with lower SES indices associated with lower language skills, and more so in children with ASD.

Conclusion: These results demonstrate that variability in EL and RL skills in young children with autism can be accounted for by socioeconomic variables. These findings highlight the necessity for targeted intervention and effective implementation strategies for children with ASD from low-resource households and communities and for policies designed to improve learning opportunities and access to services for these young children and their families.

Address for reprints: Inna Fishman, PhD, Department of Psychology, Brain Development Imaging Laboratories, San Diego State University, 6363 Alvarado Court, Suite 200, San Diego, CA 92120; ifishman@sdsu.edu.

Disclosure: The authors declare no conflict of interest.

Supplemental digital content is available for this article. Direct URL citations appear in the printed text and are provided in the HTML and PDF versions of this article on the journal's Web site (www.jdbp.org). 


\section{Keywords}

autism spectrum disorder; socioeconomic status; language development

As disorders of development, autism spectrum disorders (ASD) can have profound, life-long consequences for affected individuals and their families. Although language delays are no longer required for a diagnosis of $\mathrm{ASD},{ }^{1}$ they are extremely common in children who develop the disorder and are often the first indicator for parents or pediatricians that a child's development may not be progressing typically. ${ }^{2}$ Roughly one-quarter of individuals with ASD never acquire spoken language, whereas others can be highly verbal but may still display language abnormalities, ${ }^{3}$ including problems with pragmatic aspects of language, such as prosody and turn-taking. Overall, although language abilities vary across the spectrum, most young children with ASD are at risk for some form of language delay or speech anomaly, which can impede later academic performance, social communication, relationships, and quality of life. ${ }^{4}$

The bulk of language acquisition occurs during a sensitive period for language learning between birth and 5 years of age. Within this period, there are several stages of language development, characterized by the onset of distinct skill sets. In the toddler years, approximately between 1.5 and 2 years of age, neurotypical children experience a vocabulary "explosion," 5 and after early childhood, it becomes increasingly difficult to acquire language. Furthermore, during this early sensitive period, brain structures and functional networks subserving language are particularly vulnerable to environmental risk factors, such as impoverished language exposure, early life stress, and poor nutrition. ${ }^{6}$ Indeed, some evidence suggests that language brain circuits may be affected by child's sociodemographic circumstances more so than other neurocognitive systems. ${ }^{7}$ Given the potential benefits of early interventions, characterization of the factors that contribute to language delays in young children with ASD has the potential to inform targeted interventions for children from lower-socioeconomic status (SES) backgrounds (e.g., Ref. 8). Although early language delays can have deleterious impacts on functional outcomes, the plasticity of the developing brain presents a promising window for positively affecting this trajectory. Furthermore, identifying early predictors of risk and developing targeted interventions is particularly important because language skills in ASD may be less responsive to interventions after 6 years of age, ${ }^{9}$ and outcomes of speech or language therapy, even in the toddler years, remain highly variable. ${ }^{10}$

Critically, it remains unknown whether and to what degree socioeconomic factors commonly referred to as SES (e.g., household income and parental education level) are associated with language skills in children with ASD. The circumstances associated with SES (e.g., degree of financial stress, exposure to environmental pollution, and access to nutritious foods) can have broad-reaching impacts on clinical and developmental outcomes, particularly in the domain of language. Specifically, findings on SES disparities in linguistic skills (and literacy) are some of the most robust in the developmental literature (e.g., on high school entry, adolescents from lower-SES backgrounds perform, on average, 5 years behind their higher-SES peers on measures of literacy). ${ }^{11}$ Associations between SES and prelinguistic 
cognitive skills can be observed in typical development as early as the first year of life, with lower prelanguage skills reported in low versus mid-to-high SES female infants at 7 months of age. ${ }^{12}$ By the age of 12 months, infants from lower-SES households already show poorer developmental indices on early language measures compared with higher-SES peers, highlighting the importance of early interventions targeting literacy and language skills for low SES communities and families with young children. ${ }^{13}$ By 18 months of age, toddler vocabulary and speed and accuracy of language understanding, or language processing efficiency, are associated with family SES, ${ }^{14}$ and by 21 months of age, large differences in language and memory skills are observed based on parental education. ${ }^{15}$ The relationship between SES and language outcomes has also been reported in children who were born prematurely, with those receiving Medicaid-based insurance (indicating lower financial resources compared with individuals with private health insurance) showing lower scores on measures of both receptive language (RL) and expressive language (EL) between ages 15 and 30 months. ${ }^{13}$ This finding was attributed to multiple factors associated with SES, including the quality of the child's home language environment. In fact, language exposure in the home, or language environment, which has been operationalized as the number and complexity of words heard in the home, the number of conversational turns between caregivers and children, and the number of books in the home, ${ }^{16}$ may be a stronger predictor of child language outcomes than other SES variables. ${ }^{17,18}$

Much less is known about associations between SES and language in young children with ASD, in large part because of a dearth of research on individuals with ASD from lower-SES backgrounds. The lack of research focusing on children and adults with ASD from underresourced communities is partly related to societal challenges and barriers associated with participating in research studies (e.g., limited transportation or ability to take time off work and inadequate parenting supports). There is, however, some limited evidence suggesting that SES is associated with clinical and diagnostic outcomes in autism, ${ }^{19}$ such as symptom trajectories and developmental gains over time. Children with ASD from lower-SES families are also more likely to receive a comorbid intellectual disability diagnosis than their mid-tohigh SES peers. ${ }^{20}$ Possibly accounting for these links is the fact that lower SES is associated with later age of autism diagnosis and reduced access to intervention services, ${ }^{21}$ highlighting the significant socioeconomic disparities in ascertainment of autism.

However, none of the extant evidence on clinical outcomes associated with ASD and variable SES backgrounds directly relates to language emergence in the first years of life in autism, a critical time for language development and an optimal time for effective interventions. Given that language development is contingent on and constrained by early reciprocal social engagement, which is known to be affected from very early in life in ASD, 22 such aspects of the home language environment as reduced conversational turn-taking, lower total number of utterances, and lower number of child-directed words associated with lower SES may compound the effect of altered social engagement on language development in young children with ASD. ${ }^{17}$

In sum, understanding how experiences associated with SES factors relate to prelinguistic skills and language development in autism during early years, when children are most receptive to interventions, is critically lacking. Understanding the links between family and 
household resources and child's developmental outcomes is needed to inform interventions that may be targeted specifically to children with ASD from low-resource communities.

As such, here we focus on receptive and expressive prelinguistic and language skills as they relate to socioeconomic variability in a diverse group of young children with and without autism enrolled in a larger study examining the early behavioral and neural markers of autism. Because there is no consensus in public health research regarding the measurement of SES, ${ }^{23}$ we used several individual- and neighborhood-level SES indicators (including household income taking into account household size, maternal education, and median income based on postal code $)^{23}$ to examine the relationships between factors associated with SES and RL and EL skills. We hypothesized that socioeconomic variables would be positively associated with receptive and expressive prelinguistic and language skills in toddlers and preschoolers over and above the effect of diagnosis on language skills.

\section{METHODS}

\section{Participants}

Participants were enrolled in an ongoing longitudinal study of early brain markers of autism spectrum disorders (ASD). Children between the ages of 15 and 64 months with a (suspected) diagnosis of autism were referred to the Toddler MRI Project from specialty autism clinics, state-funded early education and developmental evaluation programs, local pediatricians, service providers, and community clinics. Typically developing (TD) children were recruited from the community, including early head start programs, and through print and social media advertisements. Participants with ASD were excluded for any comorbid neurological disorders (e.g., cerebral palsy) or syndromic forms of ASD (e.g., fragile X or Rett syndrome). Participants in the TD group were excluded for prematurity ( $<36$ weeks of gestation), family history of (first-degree relative with) ASD, intellectual disability, or other heritable psychiatric or neurological disorders. Other exclusionary criteria for all participants were history of nonfebrile seizures, perinatal central nervous system (CNS) infection or gross CNS injury, and contraindications for MRI. One hundred seven children (ASD: $n=62$ [16 females]; TD: $\mathrm{n}=45$ [21 females]) were included in the current sample (see Table 1 for details on participant characteristics). All ASD diagnoses (also referred to as clinical best estimate in children younger than age 3$)^{24}$ were established based on the DSM- 5 criteria, ${ }^{1}$ supported by the Autism Diagnostic Observation Schedule, Second Edition ${ }^{25}$ administered by research-reliable clinicians, the Autism Diagnostic Interview-Revised ${ }^{26}$ administered to caregivers of children older than 36 months, and expert clinical judgment. Informed written consent was obtained from caregivers under protocols approved by the institutional review board. This report includes cross-sectional data only.

\section{Language and Developmental Outcomes}

Participants were administered the Mullen Scales of Early Learning (MSEL) ${ }^{27}$ a clinicianadministered assessment of language, cognitive, and motor development, which yields agecorrected standardized scores. The MSEL Expressive Language (EL) and Receptive Language (RL) scales were used as the primary language outcome variables in analyses. The Vineland Adaptive Behavior Scales, Second Edition, Survey Interview, ${ }^{28}$ a semi- 
standardized caregiver interview yielding age-normed standard scores, was administered to assess the child's adaptive communication, daily living, and social and motor skills. Caregivers also completed the Child Behavior Checklist (CBCL), ${ }^{29}$ preschool form (for ages 1.5-5 years), a measure designed to assess behavioral and emotional problems across multiple clinical domains, and the Social Communication Questionnaire, Current (SCQ), ${ }^{30}$ a screener for ASD. All TD participants' scores on the CBCL Total Problems, Internalizing Problems, and Externalizing Problems subscales fell within the normal range (all T scores < 63), and no TD participants exceeded the SCQ cutoff score of 15 (all scores < 11).

\section{Socioeconomic and Sociodemographic Variables}

Household-level socioeconomic status (SES) was assessed based on the demographic information provided by participants' caregivers, including household income, the number of individuals in the household, and the highest level of education attained by either parent. Gross annual income was reported on the following scale: $<\$ 10,000, \$ 10,001$ to 20,000 , $\$ 20,001$ to $30,000, \$ 30,001$ to $40,000, \$ 40,001$ to $50,000, \$ 50,001$ to $60,000, \$ 60,001$ to $80,000, \$ 80,001$ to $100,000, \$ 100,001$ to $150,000, \$ 150,001$ to $200,000, \$ 200,001$ to 250,000 , and $>\$ 251,000$. Income measurements were converted to income-to-needs ratio (INR) to account for family size (INR was derived by dividing the household income by the federal poverty threshold defined by family size; an INR of 1 indicates living at the federal poverty line, which according to the 2019 US government official poverty definition was $\$ 25,750$ per year for a family of 4). Maternal education was rated on a 6-point scale (less than high school, completed high school, vocational or technical school, some college, completed college, and professional or doctoral training beyond college). Neighborhoodlevel $S E S$ was estimated with median income by postal code derived from publicly available census data. ${ }^{31}$ Thus, the following household- and neighborhood-level SES variables were used: Maternal Education Level (MEL), INR, and median income by postal code (ZipIncome). Seven percent of families of children with ASD and 5\% of caregivers of TD participants chose not to provide parental education or income data. Additional variables derived from the demographic information provided by parents included the child's exposure to multiple languages, including regularly hearing at home and/or speaking languages other than English. Representative of the diversity in the San Diego metropolitan area, 50\% of children with ASD and 47\% of TD children had exposure to more than 1 language.

\section{Analytic Strategy}

Multiple regression models were used with socioeconomic variables (MEL, INR, and ZipIncome) and diagnosis as explanatory variables and Mullen RL and EL scores as outcome variables, controlling for multiple language exposure and/or ethnicity when necessary. Relationships between SES variables and MSEL and Vineland RL and EL scores were examined (amounting to $3 \times 4=12$ regression models in total) with Bonferroni correction applied, yielding a corrected alpha of $0.004(0.05 / 12)$. Because, as expected with children with ASD, we observed a floor effect for language (and other developmental indices) using MSEL T-scores, separate analyses using more continuously distributed raw scores were conducted, controlling for age and diagnosis. 


\section{Covariates}

Because a large proportion of individuals in our study self-identified as Hispanic (33\%), consistent with the population demographic in San Diego, CA,${ }^{31}$ we tested for associations between ethnicity and multiple language exposure on language outcomes ${ }^{8}$ and retained these variables in the regression models when shown to be significantly associated with language outcome variables (see Supplemental Figures S3 and S4, Supplemental Digital Content 1, http://links.lww.com/JDBP/A268). In addition, given that circumstances associated with low SES can pose significant barriers to accessing health care and services, ${ }^{21}$ we tested for effects of history of interventions on language skills in the ASD group. The receipt of interventions was coded according to intensity as reported by caregivers (low: $\Delta$ hour per week, medium: between 1 and 3 hours per week, and high: $>3$ hours per week). Finally, the effects of age and gender on the language outcomes were also examined and retained in regression models when significantly associated with language outcome variables.

\section{RESULTS}

\section{Socioeconomic and Demographic Variables: Descriptive Statistics and Group Comparisons}

Reported family income for participants in the San Diego State University Toddler MRI study ranged from $<\$ 10,000$ to $>\$ 250,000$ annually. Thirty-two percent of the families reported household incomes that qualify as low income according to federal guidelines (defined as less than $80 \%$ of the median income for the region). ${ }^{31}$ Furthermore, income-toneeds ratio (INR) ranged from below the federal poverty line to greater than 10 times the poverty line (Fig. 1B). Families were from diverse communities throughout and beyond San Diego County, including neighborhoods with high density of low-income families (Fig. 1C). White children made up most of the sample (61\%); $39 \%$ of the children were non-White or of mixed racial descent (2.8\% were reported by parents as Black, $5.6 \%$ as Asian, and $30 \%$ as more than 1 race). Seven percent of participants chose not to report their racial background.

Typically developing participants (TD) and participants with autism spectrum disorders (ASD) did not differ on INR (Fig. 1E) or median income by postal code (Zip-Income; Fig. 1F); however, TD participants had significantly higher Maternal Education Level (MEL) compared with the ASD participants ( $t=3.23, p=0.002$; Fig. 1D). Given this difference in MEL, analyses using MEL as a predictor variable were also conducted with a subset of TD participants matched (at the group level) to the ASD group on MEL. Analyses with the matched TD sample yielded findings consistent with those obtained with the larger TD sample, as reported in Supplemental Materials (Appendix 1, Supplemental Digital Content 1, http://links.lww.com/JDBP/A268).

\section{Language Outcomes and Other Developmental Indices: Descriptive Statistics and Group Comparisons}

As expected, children in the ASD group obtained significantly lower T-scores on all Mullen Scales of Early Learning (MSEL) scales compared with the TD children, who on average scored within the expected range for their age (all $p$ values $<0.0001$; Table 1 ). Similarly, as expected, children with ASD showed significantly lower adaptive behavior skills (Vineland 
Adaptive Behavior Composite: $\mathrm{M}_{\mathrm{ASD}} \pm \mathrm{SD}=76.7 \pm 9.5 ; \mathrm{M}_{\mathrm{TD}} \pm \mathrm{SD}=100.1 \pm 12.8 ; t=9.2$, $p<0.001$ ) and significantly more reported behavior problems (Child Behavior Checklist Total Problems: $\left.\mathrm{M}_{\mathrm{ASD}} \pm \mathrm{SD}=63.0 \pm 11.4 ; \mathrm{M}_{\mathrm{TD}} \pm \mathrm{SD}=42.0 \pm 8.7 ; t=-8.4, p<0.001\right)$.

Because a T-score of 20 is the minimum score for the MSEL scales (representing a score of 3 or more SDs below the mean), a substantial number of participants in the ASD group received a T-score of 20 on the receptive language (RL) ( $\mathrm{n}=18$ or 29\%) and expressive language (EL) ( $\mathrm{n}=24$ or 39\%) scales. To better capture the full performance range for the children with $\mathrm{ASD}$, raw $\mathrm{RL}(\mathrm{M} \pm \mathrm{SD}=23.9 \pm 8.9$; range: $4-48)$ and $\mathrm{EL}(\mathrm{M} \pm \mathrm{SD}=21.2 \pm$ 10.2; range: 5-50) scores were used in secondary regression models, controlling for age.

\section{Associations Between Socioeconomic Status Variables and Language}

Separate regression models were used to test for significant associations between socioeconomic variables and RL and EL. Ethnicity (Hispanic, non-Hispanic) and multiple language exposure (exposure to English only and exposure to $>1$ language) were included as covariates in all analyses and were retained in the models if revealed to be significantly associated with language skills. Otherwise, socioeconomic status (SES) factors (individually) and diagnosis were the only predictors included in the models (Table 2). Because no significant associations between age or gender and RL or EL skills were detected within and across diagnostic groups, neither age nor gender was retained in final regression models (with the exception of age, which was included as a covariate in analyses using raw MSEL scores).

Maternal Education Level as related to EL (MSEL EL) was examined first, controlling for the effect of diagnosis (ASD, TD). As expected, there was a strong, significant effect of diagnosis on EL, with ASD diagnosis associated with lower EL skills $\left(\beta_{\mathrm{Dx}}=-5.7, t=-3.6\right.$, $p<0.001$, partial $r^{2}=0.11$; Table 2). Controlling for the effect of diagnosis, maternal education was significantly associated with $\mathrm{EL}\left(\beta_{\mathrm{MEL}}=2.98, t=3.8, p=0.001\right.$, partial $r^{2}=$ 0.10 ), and this association remained significant after applying a Bonferroni correction for multiple comparisons. Because maternal education and diagnosis are correlated in this sample (mothers of participants with ASD had significantly lower educational levels; Fig. 1D), we also investigated the effect of maternal education on EL using a smaller TD sample, matched to the ASD sample on MEL. The results using the matched sample were broadly the same as with the larger sample (see Supplemental Materials, Appendix 1 and Supplemental Table S1, Supplemental Digital Content 1, http://links.lww.com/JDBP/A268).

Maternal education was also positively associated with parent-rated EL skills (Vineland Expressive Language scale; $\beta_{\mathrm{MEL}}=0.61, t=2.5, p=0.01$, partial $t^{2}=0.06$; Fig. 2B), controlling for the effect of diagnosis $\left(\beta_{\mathrm{Dx}}=-1.88, t=-4.37 p<0.001\right.$, partial $r^{2}=0.17$ ), although the association was not significant after applying a Bonferroni-corrected alpha for multiple comparisons. The INR was also associated with EL (MSEL EL), controlling for the effect of diagnosis, although not significant after correcting for multiple comparisons ( $\beta_{\text {INR }}$ $=1.16, t=2.22, p=0.03$, partial $t^{2}=0.05 ; \beta_{\mathrm{Dx}}=-6.12, t=-3.54, p<0.001$, partial $t^{2}=$ 0.12 ; Fig. 2E). 
Regarding RL, maternal education was positively associated with clinician-assessed RL skills (MSEL RL: $\beta_{\mathrm{MEL}}=3.60, t=3.16, p=0.002$, partial $t^{2}=0.09$ ), controlling for the effect of diagnosis $\left(\beta_{\mathrm{Dx}}=-6.75, t=-3.30, p=0.001\right.$, partial $t^{2}=0.10$; Fig. 2C). Maternal education was also associated with parent-rated RL skills (Vineland Receptive Language: MSEL RL: $\beta_{\mathrm{MEL}}=0.85, t=2.86, p=0.005$, partial $r^{2}=0.08$ ), controlling for the effect of diagnosis $\left(\beta_{\mathrm{Dx}}=-1.78, t=-3.50 p<0.001\right.$, partial $t^{2}=0.08$; Fig. $\left.2 \mathrm{C}\right)$, although not significant after applying the Bonferroni-corrected alpha.

Multiple regression models using the MSEL EL and RL raw scores (controlling for age and diagnosis) instead of standardized T-scores yielded a similar pattern of results (see Supplemental Figure S1, Supplemental Digital Content 1, http://links.lww.com/JDBP/ A268). No significant effects of Zip-Income on children's EL and RL raw scores were detected.

\section{Socioeconomic Status, Language, and History of Interventions}

Of the participants with ASD, $60 \%$ have reported receiving some form of intervention (applied behavior analysis, speech therapy, occupational therapy, or other services). Intervention receipt and intervention intensity had no effect on any of the SES variables (MEL, INR, Zip-Income; all $p$ s > 0.2; see Figure S2a-c; S2d-f, Supplemental Digital Content 1, http://links.lww.com/JDBP/A268, respectively), nor on EL or RL scores. In addition, age when the intervention commenced was not associated with the SES variables, nor with EL or RL scores (all $p s>0.2$ ).

\section{DISCUSSION}

We investigated associations between both house-hold-level and neighborhood-level socioeconomic indices and emerging language (and prelinguistic) skills in toddlers and preschoolers with autism spectrum disorders (ASD), and their typically developing (TD) peers. We found that Maternal Education Level (MEL) accounted for variability in expressive language (EL) and receptive language (RL) skills in young children over and above the influence of autism diagnosis. Specifically, lower maternal education was associated with lower EL and RL scores. These results highlight the importance of examining patterns of language development in children with ASD from low-resource backgrounds because they may stand to benefit the most from early, targeted interventions, given that they may be more likely to experience delayed language development in early childhood.

Although ours is among the first reports demonstrating associations between socioeconomic status (SES) factors and language emergence in young children with ASD (see also a recent study ${ }^{32}$ ), these findings align with other studies reporting links between SES and clinical and diagnostic outcomes in older children with ASD. ${ }^{19,33}$ Specifically, an investigation of developmental trajectories in ASD revealed that children with highly educated mothers were more likely to have a "positive developmental trajectory" characterized by improvement in ASD symptoms over time ("blooming") compared with children of less-educated mothers. ${ }^{33}$ 
Importantly, the amount of words a child hears in the home varies widely across households and varies by SES. ${ }^{34}$ Although not directly measured in this study, 1 possible explanation for the observed associations between socioeconomic factors and language skills relates to the amount and complexity of language to which children are exposed in the home. ${ }^{32}$ Not only is language exposure critical for typical language development, but the reciprocal nature of language (i.e., contingent responsivity to child preverbal or verbal communication) is also associated with child EL skills and is a frequent target in early parent-child dyadic treatments in ASD. ${ }^{35}$ Indeed, the association between parental educational level and prelinguistic development in infants later diagnosed with ASD is mediated by the richness of the home language environment, including "hearing more adult words and experiencing more conversational turns." 32 As such, interventions that promote reciprocal communication between parents and children in the first years of life may be of particular benefit to children with ASD from low-resource communities, who may be exposed to less language in the home. ${ }^{18}$ Children with ASD from low-resource households may benefit from uniquely tailored interventions that account for and address life circumstances associated with low SES. ${ }^{8}$

In addition, the general pattern of our findings suggests that maternal educational level may be a particularly salient component of SES because it relates to early childhood language development in children with and without ASD. Among the 3 SES variables tested in our analysis, maternal educational level was the most consistently associated with language outcome variables. These findings align with others linking maternal educational level and language skills in early childhood. ${ }^{37}$ Furthermore, maternal education has been found to mediate the efficacy of early language intervention on language skills in toddlers with language delay. ${ }^{36}$ This relationship may be mediated by the home language environment, which —although not directly measured in this study—has a strong relationship with maternal educational level (as reviewed in Ref. 37). The results of this study need to be interpreted in the context of several limitations. First, SES is notoriously difficult to operationalize and measure because socioeconomic variables serve as a proxy for a multitude of factors and quality of life attributes, as well as the opportunities and privileges afforded to people within society, that may all meaningfully affect early child development. 38 Although we implemented several distinct measures to quantify SES at both household and neighborhood levels, there are many other possible SES indicators that were not included in this study. In addition, our conclusions may be limited by a fairly modest sample size and the initial study design, which prevent us from examining more complex mediation models of how SES variables relate to language development to explore what may account for the observed associations between SES variables (primarily maternal education) and language skills in early childhood. A further limitation of this study is the lack of measures characterizing the home language environment, which has been shown to mediate the effect of maternal education on language skills in young children with ASD. ${ }^{32}$

Finally, perhaps because of the differences in recruitment avenues for TD children versus children with ASD, mothers of our TD participants had significantly higher education levels than those of participants with ASD. As such, diagnosis and maternal education were correlated in our sample, thereby introducing increased susceptibility for error in the regression models, including both diagnosis and MELs. We addressed the significant 
differences in MELs between our TD and ASD samples by conducting secondary regression models using a smaller TD subsample, matched to the ASD group on maternal education, as a sensitivity analysis. The results from regression models on the matched sample yielded very similar findings.

Overall, the results from this study demonstrate that variability in EL and RL skills in young children with and without autism can be accounted for by socioeconomic variables. These findings highlight the necessity for targeted intervention and effective implementation strategies for children with ASD from low-resource households and communities and for policies designed to improve learning opportunities and access to services for these young children and their families. To further develop and implement intervention strategies for lower-resourced families of children with ASD, community-partnered approaches to understanding the needs of these children and their families may be of particular benefit. ${ }^{39}$ The findings also demonstrate that the field of autism research and practice stands to benefit from increased attention to sociodemographic variables as they relate to language, clinical, and cognitive outcomes in ASD because individuals and families with limited access to financial and social resources traditionally underrepresented in research may add unique aspects to our understanding of development in autism and may benefit the most from these efforts. ${ }^{19}$

\title{
Supplementary Material
}

Refer to Web version on PubMed Central for supplementary material.

\section{ACKNOWLEDGMENTS}

\begin{abstract}
The authors are grateful to Chris Fong, MA, and Lisa Mash, MS, for invaluable assistance with data collection and Georg Matt, PhD, for statistical consultation. The authors also thank Themba Carr, $\mathrm{PhD}$, for insightful discussions on community-centric research and practice. The authors' strongest gratitude goes to the children and families who so generously dedicated their time and effort to this research.
\end{abstract}

Supported by grants from the National Institutes of Health (R01 MH107802 to I. Fishman) and Autism Speaks (predoctoral fellowship to L. Olson).

\section{REFERENCES}

1. Diagnostic and Statistical Manual of Mental Disorders. 5th ed. Washington, DC: American Psychiatric Association; 2013.

2. Richards M, Mossey J, Robins DL. Parents' concerns as they relate to their child's development and later diagnosis of autism spectrum disorder. J Dev Behav Pediatr. 2016;37:532-540. [PubMed: 27541581]

3. Tager-Flusberg H, Kasari C. Minimally verbal school-aged children with autism spectrum disorder: the neglected end of the spectrum. Autism Res. 2013;6:468-478. [PubMed: 24124067]

4. Petersen IT, Bates JE, D'Onofrio BM, et al. Language ability predicts the development of behavior problems in children. J Abnorm Psychol. 2013;122:542-557. [PubMed: 23713507]

5. McMurray B Defusing the childhood vocabulary explosion. Science. 2007;317:631. [PubMed: 17673655]

6. Kuhl PK. Early language learning and literacy: neuroscience implications for education. Mind Brain Educ. 2011;5:128-142. [PubMed: 21892359]

7. Hackman DA, Farah MJ, Meaney MJ. Socioeconomic status and the brain: mechanistic insights from human and animal research. Nat Rev Neurosci. 2010;11:651-659. [PubMed: 20725096] 
8. Carr T, Shih W, Lawton K, et al. The relationship between treatment attendance, adherence, and outcome in a caregiver-mediated intervention for low-resourced families of young children with autism spectrum disorder. Autism. 2016;20:643-652. [PubMed: 26290524]

9. Pickles A, Anderson DK, Lord C. Heterogeneity and plasticity in the development of language: a 17-year follow-up of children referred early for possible autism. J Child Psychol Psychiatry. 2014;55:1354-1362. [PubMed: 24889883]

10. Pringle B, Colpe LJ, Blumberg SJ, et al. Diagnostic history and treatment of school-aged children with autism spectrum disorder and special health care needs. NCHS Data Brief. 2012;1-8.

11. Reardon S, Valentino R, Kalogrides D, et al. Patterns and trends in racial academic achievement gaps among states, 1999-2011 ICenter for Education Policy Analysis Available at: https:// cepa.stanford.edu/content/patterns-and-trends-racial-academic-achievement-gaps-amongstates-1999-2011. Published 2013 Accessed June 7, 2018.

12. Betancourt LM, Brodsky NL, Hurt H. Socioeconomic (SES) differences in language are evident in female infants at 7 months of age. Early Hum Dev. 2015;91:719-724. [PubMed: 26371987]

13. Wild KT, Betancourt LM, Brodsky NL, et al. The effect of socioeconomic status on the language outcome of preterm infants at toddler age. Early Hum Dev. 2013;89:743-746. [PubMed: 23803578]

14. Fernald A, Marchman VA, Weisleder A. SES differences in language processing skill and vocabulary are evident at 18 months. Dev Sci. 2013;16:234-248. [PubMed: 23432833]

15. Noble KG, Engelhardt LE, Brito NH, et al. Socioeconomic disparities in neurocognitive development in the first two years of life. Dev Psychobiol. 2015;57:535-551. [PubMed: 25828052]

16. Gilkerson J, Richards JA, Warren SF, et al. Mapping the early language environment using all-day recordings and automated analysis. Am J Speech Lang Pathol. 2017;26:248-265. [PubMed: 28418456]

17. Hoff E The specificity of environmental influence: socioeconomic status affects early vocabulary development via maternal speech. Child Dev. 2003;74:1368-1378. [PubMed: 14552403]

18. Hart B, Risley TR. Meaningful Differences in the Everyday Experience of Young American Children. Baltimore, MD: Brookes; 1995.

19. Baio J, Wiggins L, Christensen DL, et al. Prevalence of autism spectrum disorder among children aged 8 years-autism and developmental disabilities monitoring network, 11 sites, United States, 2014. MMWR Surveill Summ. 2018;67:1-23.

20. Levaot Y, Meiri G, Dinstein I, et al. Autism prevalence and severity in Bedouin-Arab and Jewish communities in Southern Israel. Community Ment Health J. 2019;55:156-160. [PubMed: 29388003]

21. Dickerson AS, Rahbar MH, Pearson DA, et al. Autism spectrum disorder reporting in lower socioeconomic neighborhoods. Autism. 2016;21:470-480. [PubMed: 27627912]

22. Klin A, Shultz S, Jones W. Social visual engagement in infants and toddlers with autism: early developmental transitions and a model of pathogenesis. Neurosci Biobehav Rev. 2015;50:189-203. [PubMed: 25445180]

23. Oakes JM, Rossi PH. The measurement of SES in health research: current practice and steps toward a new approach. Soc Sci Med. 2003;56:769-784. [PubMed: 12560010]

24. Ozonoff S, Young GS, Landa RJ, et al. Diagnostic stability in young children at risk for autism spectrum disorder: a baby siblings research consortium study. J Child Psychol Psychiatry. 2015;56: 988-998. [PubMed: 25921776]

25. Lord C, Rutter M, Dilavore P, et al. Autism Diagnostic Observation Schedule, Second Edition (ADOS-2) Manual (Part I): Modules 1-4. Torrance, CA: Western Psychological Services; 2012.

26. Lord C, Rutter M, Le Couteur A. Autism Diagnostic Interview-Revised: a revised version of a diagnostic interview for caregivers of individuals with possible pervasive developmental disorders. J Autism Dev Disord. 1994;24:659-685. [PubMed: 7814313]

27. Mullen E Mullen Scales of Early Learning Manual. Circle Pines, MN: American Guidance Services, Inc.; 1995.

28. Sparrow S, Cicchetti D, Balla D. Vineland Adaptive Behavior Scales, 2nd ed. New York, NY: Pearson Clinical; 2005. 
29. Achenbach T, Rescorla L. Child Behavior Checklist. Burlington, VT: University of Vermont, Research Center for Children, Youth \& Families; 2000.

30. Rutter M, Bailey A, Lord C. Social Communication Questionnaire (SCQ). Los Angeles, CA: Western Psychological Services; 2003.

31. 2013-2017 American Community Survey 5-Year Estimates. U.S. Census Bureau Available at: https://factfinder.census.gov/. Published 2017 Accessed June 7, 2018.

32. Swanson MR, Donovan K, Paterson S, et al. Early language exposure supports later language skills in infants with and without autism. Autism Res. 2019;12:1784-1795. [PubMed: 31254329]

33. Fountain C, Winter AS, Bearman PS. Six developmental trajectories characterize children with autism. Pediatrics. 2012;129:e1112-e1120. [PubMed: 22473372]

34. Hoff E How social contexts support and shape language development. Dev Rev. 2006;26:55-88.

35. Ingersoll B, Wainer A. Initial efficacy of Project ImPACT: a parent-mediated social communication intervention for young children with ASD. J Autism Dev Disord. 2013;43:2943-2952. [PubMed: 23689760]

36. Yoder PJ, Warren SF. Relative treatment effects of two prelinguistic communication interventions on language development in toddlers with developmental delays vary by maternal characteristics. J Speech, Lang Hear Res. 2001;44:224-237. [PubMed: 11218105]

37. Conant LL, Liebenthal E, Desai A, et al. The relationship between maternal education and the neural substrates of phoneme perception in children: interactions between socioeconomic status and proficiency level. Brain Lang. 2017;171:14-22. [PubMed: 28437659]

38. Shavers VL. Measurement of socioeconomic status in health disparities research. J Natl Med Assoc. 2007;99:1013-1023. [PubMed: 17913111]

39. Carr T, Lord C. A pilot study promoting participation of families with limited resources in early autism intervention. Res Autism Spectr Disord. 2016;25:87-96. 
A

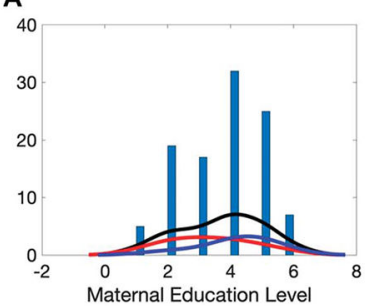

D

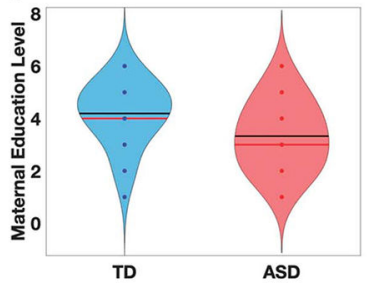

B.

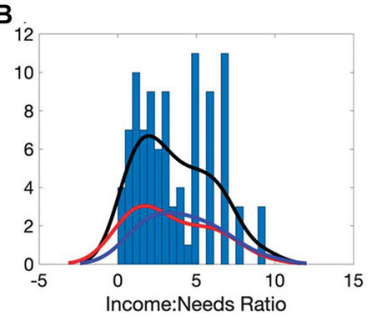

E

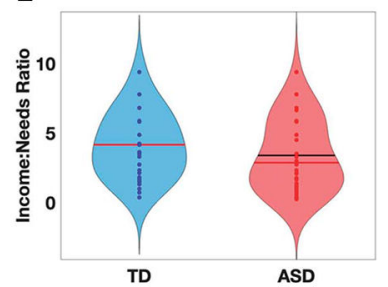

C

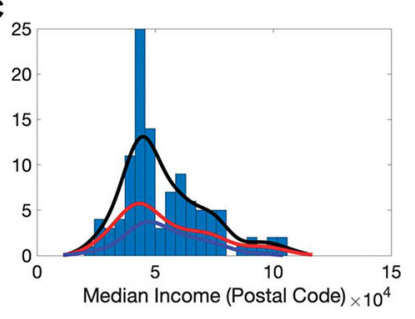

F

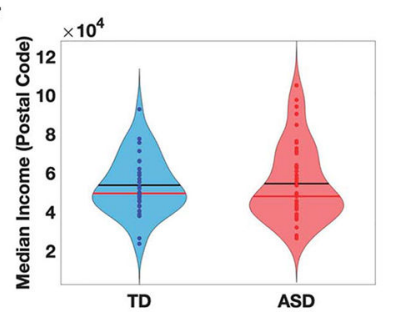

Figure 1.

Distributions of socioeconomic variables. Frequency distribution of (A) MEL, (B) INR (household income adjusted by family size as a ratio of federal poverty threshold; an INR of one indicates living at the poverty threshold), and (C) median income (postal code based). A-C, The distribution curve for ASD, TD and combined ASD + TD groups is represented with red, blue, and black lines, respectively. D-F, Comparisons of (D) maternal education, (E) INR, and (F) median income (postal-code based) in TD and ASD groups. MEL among ASD participants was significantly lower than that of TD participants $\left(t_{(104)}=3.23, p<\right.$ 0.002). ASD, autism spectrum disorders; INR, income-to-needs ratio; MEL, Maternal Education Level; TD, typically developing. 
A

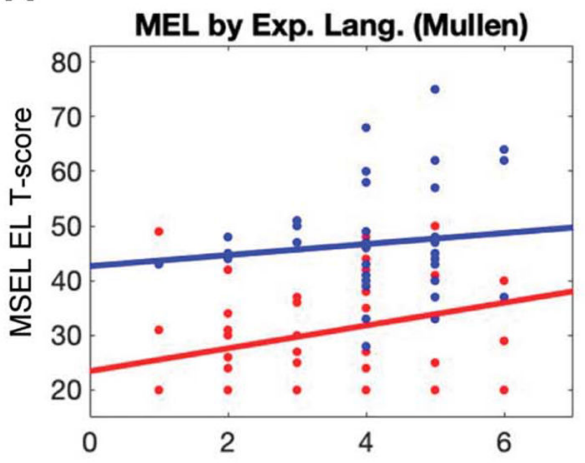

C

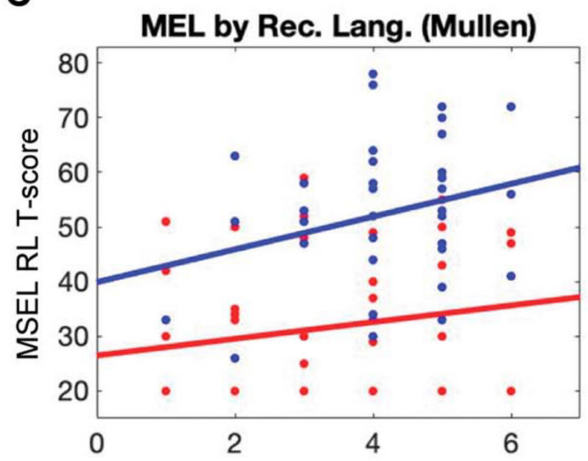

B

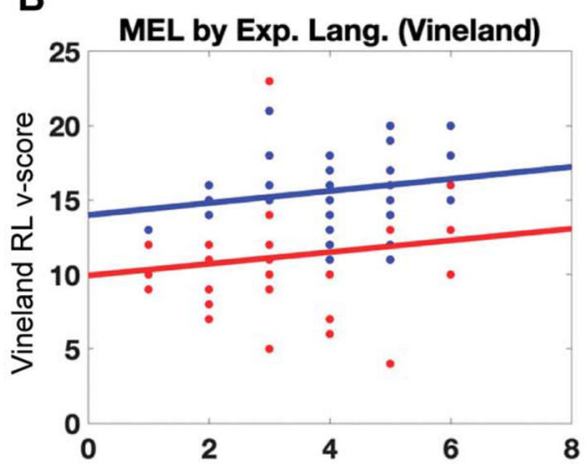

D

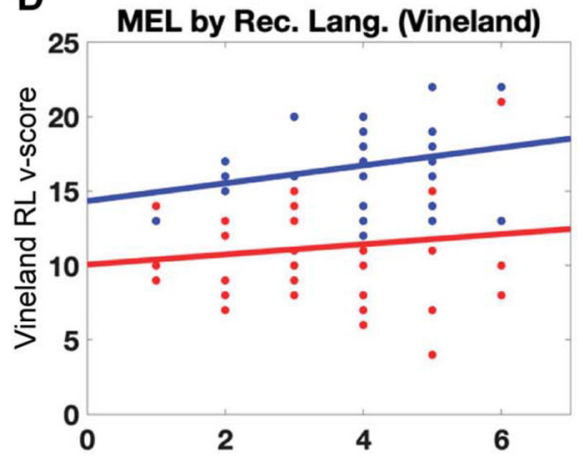

TD

E

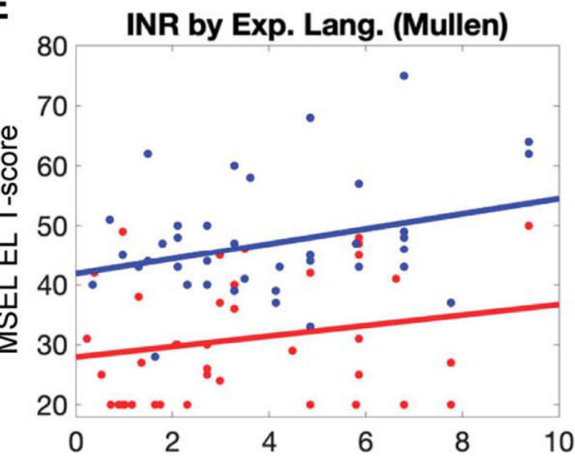

- ASD

Figure 2.

Associations between socioeconomic variables and RL and EL. A-D, Association between MEL and (A) EL skills as measured with MSEL EL, (B) parent-rated EL skills as reported on the Vineland Adaptive Behavior Scales, (C) RL as measured with MSEL RL, and (D) parent-rated RL as reported on the Vineland Adaptive Behavior Scales. E, Association between INR and EL skills as measured with MSEL EL. ASD, autism spectrum disorders; EL, expressive language; INR, income-to-needs ratio; MEL, Maternal Education Level; MSEL, Mullen Scales of Early Learning; RL, receptive language; TD, typically developing. 


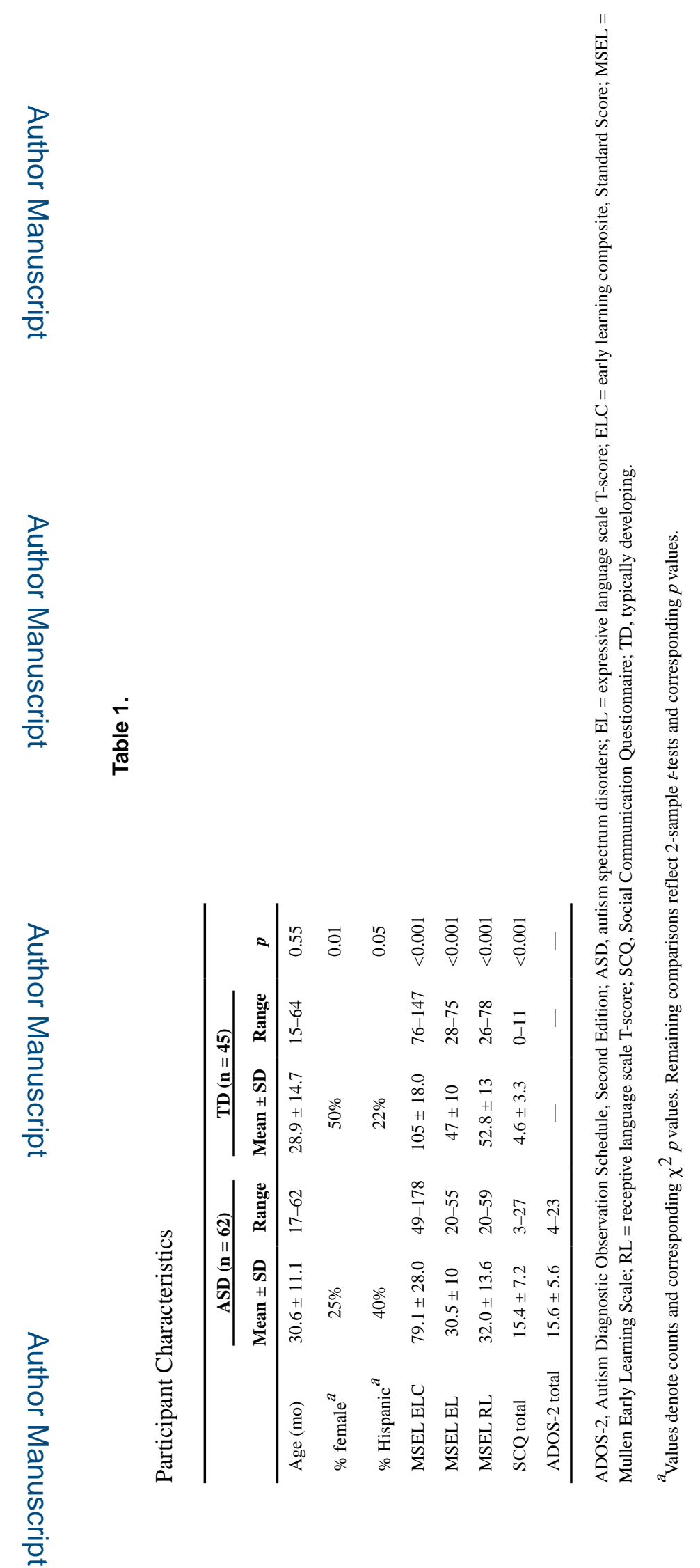

J Dev Behav Pediatr. Author manuscript; available in PMC 2021 February 05. 


\section{을 \\ ํㅗㄹ}



永

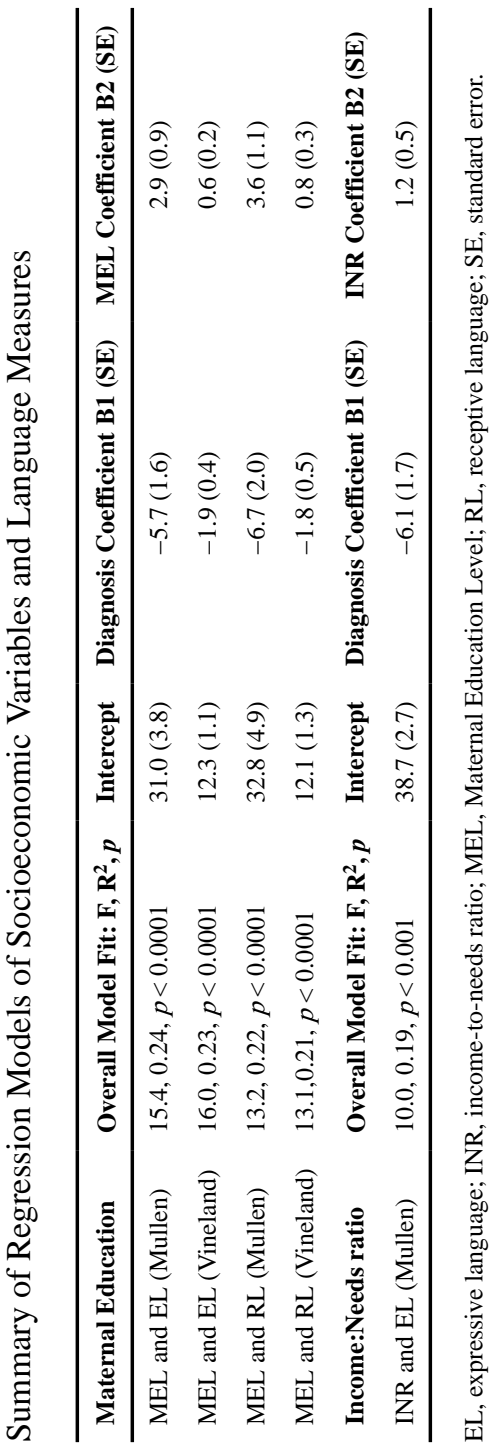

J Dev Behav Pediatr. Author manuscript; available in PMC 2021 February 05. 